\title{
Estado nutricional de idosos residentes nos municípios da Superintendência Regional de Saúde de Uberaba: estudo transversal
}

\author{
Nutritional status of elderly residents in the municipalities of the Regional \\ Healthcare Administration of Uberaba: a cross-sectional study
}

\author{
Renata Damião ${ }^{1}$, Joilson Meneguci², Álvaro da Silva Santos ${ }^{3}$, \\ Alicia Matijasevich ${ }^{4}$, Paulo Rossi Menezes ${ }^{4}$
}

Damião R, Meneguci J, Santos AS, Matijasevich A, Menezes PR. Estado Nutricional de idosos residentes nos municípios da Superintendência Regional de Saúde de Uberaba: estudo transversal / Nutritional status of elderly residents in the municipalities of the Regional Healthcare Administration of Uberaba: a cross-sectional study. Rev Med (São Paulo). 2019 set.-out.;98(5):290-7.

\begin{abstract}
RESUMO: Objetivo: avaliar o estado nutricional de idosos residentes nos municípios da Superintendência Regional de Saúde de Uberaba. Material e Métodos: Estudo transversal, realizado com indivíduos com $\geq 60$ anos de idade, residentes em Minas Gerais, Brasil. O estado nutricional foi avaliado pela Mini Avaliação Nutricional (MAN). Calcularam-se as distribuições de frequências relativas e absolutas das variáveis estudadas. As diferenças encontradas entre as prevalências do estado nutricional e os componentes da MAN segundo sexo, foram testadas utilizando-se a estatística do qui-quadrado. Resultados: Foram analisados 3.101 idosos. A prevalência de desnutrição entre os idosos foi de $1,7 \%$ e, $27,8 \%$ de risco de desnutrição. Observouse diferenças estatisticamente significantes no estado nutricional entre os sexos, principalmente em relação ao risco de desnutrição, sendo $24,4 \%$ nos homens e $29,9 \%$ nas mulheres. Nos parâmetros de diagnóstico do estado nutricional entre os idosos desnutridos e aqueles em risco de desnutrição, a redução do perímetro da panturrilha, perda de peso, diminuição da ingestão alimentar e o não consumo de frutas e vegetais apresentaram maiores percentuais. Os idosos desnutridos, em ambos os sexos, apresentaram piores parâmetros antropométricos, de saúde, alimentares e de auto avaliação na percepção da saúde e do estado nutricional quando comparados com idosos sob risco de desnutrição. Conclusão: O elevado déficit do estado nutricional, desnutrição e risco de desnutrição, observado nos idosos residentes na comunidade dos municípios avaliados demonstra um problema de saúde pública. Espera-se que os achados desse estudo chamem a atenção de setores que trabalham com a integralidade da saúde dos idosos.
\end{abstract}

Descritores: Estado nutricional; Idoso; Serviços de saúde para idosos; Saúde do idoso.

\begin{abstract}
Objective: to evaluate the nutritional status of elderly residents in the municipalities of the Regional Healthcare Administration of Uberaba. Material and methods: Cross-sectional study, conducted with individuals with $\geq 60$ years of age, residing in Minas Gerais, Brazil. Nutritional status was evaluated by the Mini Nutritional Assessment (MNA). The distributions were calculated relative and absolute frequencies of the variables studied. The differences found between the prevalence of nutritional status and components of MNA according to sex were tested using the Chi-square statistic. Results: It is analyzed 3,101 elderly. The prevalence of malnutrition among seniors was $1.7 \%$ and $27.8 \%$ risk of malnutrition. Observed statistically significant differences in nutritional status between the sexes, particularly in relation to the risk of malnutrition, being $24.4 \%$ in men and $29.9 \%$ in women. In the diagnosis of nutritional status among the elderly, malnourished and those at risk of malnutrition, reducing the calf girth, weight loss, decreased food intake and the consumption of fruit and vegetables not presented higher percentages. The elderly, malnourished, in both sexes, showed worse health, anthropometric parameters and selfevaluation in the perception of health and nutritional status when compared with elderly people at risk of malnutrition. Conclusion: The high deficit of nutritional status, malnutrition and risk of malnutrition observed in elderly residents in the community of the municipalities evaluated, demonstrates a public health problem. It is hoped that the findings of this study will draw the attention of sectors that work with the integral health of the elderly.
\end{abstract}

Keywords: Nutritional status; Aged; Health services for the aged; Health of the elderly.

Financiamento: Artigo extraído do Projeto intitulado: "Perfil de Saúde da População Idosa dos Municipios da Gerência Regional de Saúde - Uberaba/Minas Gerais”, financiado pela Fundação de Apoio à Pesquisa de Minas Gerais (FAPEMIG) sob número: APQ-00995-11.

1. Departamento de Nutrição, Programa de Pós-Graduação em Educação Física, Universidade Federal do Triângulo Mineiro, Tutunas, Uberaba, MG. ORCID: https://orcid.org/0000-0001-5563-7349. E-mail: renata.damiao@uftm.edu.br.

2. Programa de Pós-Graduação em Educação Física, Universidade Federal do Triângulo Mineiro, Tutunas, Uberaba, MG. ORCID: https://orcid.org/0000-00032268-3589. E-mail: joilson.meneguci@uftm.edu.br.

3. Departamento de Enfermagem em Educação e Saúde Comunitária, Programa de Pós-Graduação em Atenção à Saúde, Programa de Pós-Graduação em Psicologia, Universidade Federal do Triângulo Mineiro, Manuel Terra, Uberaba, MG. ORCID: https://orcid.org/0000-0002-8698-5650. E-mail: alvaroenf@hotmail.com.

4. Departamento de Medicina Preventiva, Faculdade de Medicina FMUSP, Universidade de São Paulo, São Paulo, SP. ORCID: Matijasevich A - https://orcid. org/0000-0003-0060-1589; Menezes PR - https://orcid.org/0000-0001-6330-3314.E-mail: alicia.matijasevich@usp.br, pmenezes@usp.br.

Endereço para correspondência: Renata Damião. Programa de Pós-Graduação em Educação Física, Universidade Federal do Triângulo Mineiro. Av. Tutunas, 490. Uberaba, MG, Brasil. CEP: 38061-500. E-mail: renata.damiao@uftm.edu.br. 


\section{INTRODUÇÃO}

$\mathrm{D}$ iante das transformações demográficas iniciadas no último século, observa-se uma população cada vez mais envelhecida na maioria dos países, inclusive no Brasil ${ }^{1,2}$. Os indivíduos idosos são mais vulneráveis ao comprometimento do estado nutricional, pois estão sujeitos a vários fatores inerentes ao processo de envelhecimento tais como problemas relacionados com a mastigação, deglutição, digestão e absorção. ${ }^{3}$ Além disso, este processo pode ser agravado pela presença de doenças crônicas e incapacidades que são frequentes nessa fase da $\operatorname{vida}^{4-6}$. As condições socioeconômicas também tem sido apontadas por agravar este quadro, pois são capazes de influenciar no acesso aos alimentos indispensáveis por fornecer os nutrientes essenciais para manutenção da saúde dos idosos ${ }^{7,8}$.

$\mathrm{O}$ déficit nutricional tem sido demonstrado em alguns estudos realizados com idosos residentes na comunidade ${ }^{9,10}$. Porém, a avaliação do estado nutricional deve ser ampla e decorrente da complexidade para se chegar a um diagnóstico nutricional individual, posto que há necessidade de realização de exames clínicos e laboratoriais, muitas vezes inviáveis para a população. Assim, tem-se vários instrumentos desenvolvidos e utilizados para este diagnóstico, sendo o mais utilizado a Mini Avaliação Nutricional, considerada uma ferramenta validada, altamente sensível (96\%) e específica (98\%) para avaliar o estado nutricional de idosos ${ }^{11,12}$.

A detecção do déficit nutricional de idosos residentes na comunidade é de suma importância, pois auxilia tanto no monitoramento do estado nutricional bem como na estruturação de intervenções e de políticas públicas específicas para este grupo etário. Portanto, este estudo teve por objetivo avaliar o estado nutricional de idosos residentes nos municípios da Superintendência Regional de Saúde de Uberaba.

\section{MATERIAL E MÉTODOS}

\section{Desenho e População de Estudo}

Estudo transversal, integrante da pesquisa "Perfil de saúde da população idosa dos municípios da Gerência Regional de Saúde - Uberaba/Minas Gerais", que teve como objetivo investigar o perfil sociodemográfico e a saúde de idosos residentes em 27 municípios, baseado na população de 80.134 indivíduos com 60 anos ou mais de idade ${ }^{2}$. Para o cálculo amostral considerou-se como referencial a população de idosos de cada município ${ }^{2}$, com os seguintes parâmetros: erro amostral de 5\%; intervalo de confiança de $95 \%$ e proporção populacional dos idosos de cada município (população idosa do município dividida pela população total do município). Assim, o cálculo da amostra mínima considerando a somatória das amostras mínimas de todos os municípios resultou em 3.513 idosos. Por meio das Secretárias Municipais de Saúde, todos os indivíduos com idade igual ou acima de 60 anos cadastrados na Estratégia de Saúde da Família e residentes na Zona Urbana foram convidados a comparecer nas Unidades de Atenção Primária à Saúde dos municípios para a realização das avaliações. Para o presente estudo foram avaliados 24 municípios.

Os critérios para inclusão dos idosos foram: atingir a pontuação mínima de acordo com o grau de escolaridade no Mini Exame do Estado Mental. ${ }^{13,14}$ Os critérios de exclusão foram: estar acamado, ser dependente de cadeira de rodas, ser portador de deficiência auditiva ou visual grave com dificuldade de comunicação.

\section{Coleta de Dados}

Para a coleta de dados foi utilizado um questionário multidimensional aplicado individualmente por entrevistadores treinados, após assinatura do Termo de Consentimento Livre e Esclarecido a qual ocorreu entre maio de 2012 a abril de 2013.

Para mensurar a massa corporal $(\mathrm{kg})$ foi utilizada uma balança eletrônica portátil, tipo plataforma, da marca Filizola, com capacidade para $150 \mathrm{~kg}$ e sensibilidade de $50 \mathrm{~g}$, na qual o indivíduo subiu descalço e usando roupas leves. Para a medida da estatura $(\mathrm{m})$ foi utilizado um estadiômetro portátil (trena de metal acondicionada em um estojo de $15 \times 15 \mathrm{~cm}$, de $2 \mathrm{~m}$ e graduado), no qual o indivíduo subiu descalço e foi posicionado com a cabeça, nádegas e calcanhares alinhados e encostados no aparelho, com a cabeça no plano horizontal de Frankfurt.

$\mathrm{O}$ índice de massa corporal (IMC) calculado com base na divisão do peso em quilogramas $(\mathrm{kg})$ pela altura em metro (m) elevada ao quadrado $(\mathrm{kg} / \mathrm{m} 2)$. Para as medidas das circunferências foi utilizada uma fita métrica inelástica e flexível, com extensão de $2 \mathrm{~m}$, dividida em cm e subdividida em $\mathrm{mm}$. A circunferência da panturrilha foi obtida pelo maior perímetro da panturrilha entre o tornozelo e o joelho, estando este dobrado em um ângulo de $90^{\circ}$. Para a circunferência do braço, o indivíduo permaneceu com o mesmo flexionado em um ângulo de $90^{\circ}$ para a marcação de um ponto médio entre o acrômio e o olecrano. Em seguida, foi solicitado que o braço ficasse estendido naturalmente ao lado do corpo para contornar a fita no ponto marcado de forma ajustada, evitando compressão da pele ou espaço entre a fita e o braço. As avaliações antropométricas foram mensuradas por antropometristas previamente treinados por um único supervisor nutricionista, para seguirem os critérios propostos por Lohman et al. ${ }^{15}$.

\section{Avaliação do Estado Nutricional}

O estado nutricional foi avaliado pela Mini Avaliação Nutricional (MAN), traduzida para a língua 
portuguesa ${ }^{16}$. A MAN compreende 18 perguntas agrupadas em cinco seções: 1) antropométrica (peso, altura, perda de peso, circunferência do braço e da panturrilha); 2) saúde (perda de peso nos últimos meses, uso de medicamentos, mobilidade, presença de lesões de pele ou escaras, problemas neurológicos, ocorrência de estresse / doença aguda nos últimos meses); 3) alimentar (número de refeições, ingestão de alimentos, consumo de líquidos, modo de se alimentar); 4) auto avaliação (percepção da saúde e do estado nutricional, ingestão alimentar); 5) tipo de moradia. Cada resposta recebeu um valor, os quais foram somados perfazendo um escore final. Um escore total $\geq 24$ indicou eutrofia, de 17 a 23,5 risco de desnutrição e $<17$ desnutrição.

\section{Análise dos dados}

Os dados coletados passaram por um processo de dupla digitação em planilha do programa Microsoft Office 2007 Excel $^{\circledR}$ e em seguida foi realizada a consistência dos dados. As diferenças encontradas entre as prevalências do estado nutricional e os componentes da MAN segundo sexo, foram testadas utilizando-se a estatística do quiquadrado. As análises estatísticas foram realizadas no software Stata $^{\circledR}$ versão 11.0. O valor de $\mathrm{p}<0,05$ foi adotado como significante.

\section{Aspectos Éticos}

Tabela 1 - Prevalência (\%) do estado nutricional de idosos segundo sexo e faixa etária. Superintendência Regional de Saúde de Uberaba, 2013

\begin{tabular}{|c|c|c|c|c|}
\hline Estado Nutricional & $\begin{array}{c}\text { Total } \\
\text { n (\%) }\end{array}$ & $\begin{array}{c}\text { Masculino } \\
\text { n (\%) }\end{array}$ & $\begin{array}{c}\text { Feminino } \\
\text { n (\%) }\end{array}$ & $\mathbf{p}^{*}$ \\
\hline Normal & $2185(70,5)$ & $888(74,2)$ & $1297(68,2)$ & 0,001 \\
\hline Risco de desnutrição & $862(27,8)$ & $292(24,4)$ & $570(29,9)$ & \\
\hline \multirow[t]{3}{*}{ Desnutrição } & $54(1,7)$ & $17(1,4)$ & $37(1,9)$ & \\
\hline & \multicolumn{4}{|c|}{ Faixa etária } \\
\hline & $\begin{array}{c}60-69,9 \text { anos } \\
n(\%) \\
\end{array}$ & $\begin{array}{c}70-79,9 \text { anos } \\
\text { n }(\%) \\
\end{array}$ & $\begin{array}{c}\geq 80 \text { anos } \\
n(\%)\end{array}$ & \\
\hline \multicolumn{5}{|l|}{ Sexo Masculino } \\
\hline Normal & $434(76,4)$ & $345(74,0)$ & $109(66,9)$ & 0,001 \\
\hline Risco de desnutrição & $130(22,9)$ & $114(24,5)$ & $48(29,4)$ & \\
\hline Desnutrição & $4(0,7)$ & $7(1,5)$ & $6(3,7)$ & \\
\hline \multicolumn{5}{|l|}{ Sexo Feminino } \\
\hline Normal & $760(71,4)$ & $417(65,3)$ & $120(60,0)$ & 0,007 \\
\hline Risco de desnutrição & $287(26,9)$ & $207(32,4)$ & $76(38,0)$ & \\
\hline Desnutrição & $18(1,7)$ & $15(2,3)$ & $4(2,0)$ & \\
\hline
\end{tabular}

*Teste qui-quadrado.

Ao comparar os idosos, os desnutridos apresentaram percentuais menores do perímetro braquial (<21 cm, 29,4\% vs 7,9\%), perímetro da panturrilha (< $31 \mathrm{~cm}, 82,4 \%$ vs 46,2\%), índice de massa corporal $(<$ $19 \mathrm{~kg} / \mathrm{m}^{2}, 41,1 \%$ vs $17,8 \%$ ), perda de peso nos últimos
Esta pesquisa seguiu os princípios éticos presentes na Declaração de Helsinque. Os protocolos de pesquisa foram avaliados e aprovados pelos Comitê de Ética em Pesquisa com Seres Humanos da Universidade Federal do Triângulo Mineiro (Parecer no 1640/2010) e Comitê de Ética em Pesquisa da Faculdade de Medicina da Universidade de São Paulo (Parecer no 083/15).

\section{RESULTADOS}

Do total de 3.430 idosos que participaram da pesquisa, 3.101(90,4\%) continham todas as informações para a presente análise, sendo 38,7\% homens e $61,3 \%$ mulheres. Ao analisar as características gerais dos idosos, observou-se predominância da faixa etária entre 60 a 69 anos, 1 a 4 anos de escolaridade, cor da pele branca, renda familiar entre $>1 \leq 3$ salários mínimos e frequência de 2 a 3 doenças crônicas.

A prevalência de desnutrição entre os idosos foi de $1,7 \%$ e $27,8 \%$ de risco de desnutrição. Observouse diferenças estatisticamente significantes no estado nutricional entre os sexos, principalmente em relação ao risco de desnutrição, sendo $24,4 \%$ nos homens e $29,9 \%$ nas mulheres. A prevalência de desnutrição e risco de desnutrição nos idosos aumentou de acordo com as faixas etárias (Tabela 1). 
Em relação as idosas as desnutridas apresentaram menores percentuais quando comparadas com as sob risco de desnutrição em relação ao perímetro braquial $(<21 \mathrm{~cm}$, $21,6 \%$ vs $5,8 \%)$, perímetro da panturrilha $(<31 \mathrm{~cm}, 75,7 \%$ vs $37,9 \%)$, índice de massa corporal $\left(<19 \mathrm{~kg} / \mathrm{m}^{2}, 40,6 \%\right.$ vs 7,9\%), perda de peso (superior a três quilos, $73,0 \%$ vs 30,7\%), mobilidade (deambula mas não sai de casa, $27,0 \%$ vs 7,1\%), problemas neuropsicológicos (demência ligeira/depressão grave, $40,6 \%$ vs 9,7\%) e ocorrência de estresse psicológico/doença aguda (sim, $86,5 \%$ vs 43,5\%) (Tabela 2).

Tabela 2 - Número e porcentagem de idosos segundo variáveis antropométricas e de saúde avaliadas pela Mini Avaliação Nutricional por sexo e estado nutricional. Superintendência Regional de Saúde de Uberaba, 2013

\begin{tabular}{|c|c|c|c|c|c|c|}
\hline \multirow[b]{2}{*}{ Variáveis } & \multicolumn{2}{|c|}{ Homens } & \multicolumn{4}{|c|}{ Mulheres } \\
\hline & $\begin{array}{c}\text { Desnutrição } \\
\text { n (\%) }\end{array}$ & $\begin{array}{l}\text { Risco de desnutrição } \\
\text { (n (\%) }\end{array}$ & $\mathbf{p}^{*}$ & $\begin{array}{c}\text { Desnutrição } \\
n(\%)\end{array}$ & $\begin{array}{c}\text { Risco de desnutrição } \\
\mathrm{n}(\%)\end{array}$ & $\mathbf{p}^{*}$ \\
\hline \multicolumn{7}{|l|}{ Antropométricas } \\
\hline \multicolumn{7}{|l|}{ Perímetro braquial (cm) } \\
\hline$<21$ & $5(29,4)$ & $23(7,9)$ & & $8(21,6)$ & $33(5,8)$ & \\
\hline$\geq 21 \leq 22$ & $5(29,4)$ & $34(11,7)$ & $<0,001$ & $10(27,0)$ & $41(7,2)$ & $<0,001$ \\
\hline$>22$ & $7(41,2)$ & $235(80,4)$ & & $19(51,4)$ & $496(87,0)$ & \\
\hline \multicolumn{7}{|l|}{ Perímetro da } \\
\hline panturrilha $(\mathrm{cm})$ & & & 0,004 & & & $<0,001$ \\
\hline$<31$ & $14(82,4)$ & $135(46,2)$ & & $28(75,7)$ & $216(37,9)$ & \\
\hline$\geq 31$ & $3(17,6)$ & $157(53,8)$ & & $9(24,3)$ & $354(62,1)$ & \\
\hline \multicolumn{7}{|l|}{ Índice de massa } \\
\hline corporal $\left(\mathrm{kg} / \mathrm{m}^{2}\right)$ & & & 0,011 & & & $<0,001$ \\
\hline$<19$ & $7(41,1)$ & $52(17,8)$ & & $15(40,6)$ & $45(7,9)$ & \\
\hline$\leq 19<21$ & $6(35,3)$ & $56(19,2)$ & & $6(16,2)$ & $51(8,9)$ & \\
\hline$\leq 21<23$ & $2(11,8)$ & $51(17,5)$ & & $9(24,3)$ & $81(14,3)$ & \\
\hline$\geq 23$ & $2(11,8)$ & $133(45,5)$ & & $7(18,9)$ & $393(68,9)$ & \\
\hline \multicolumn{7}{|l|}{ Saúde } \\
\hline Perda de peso & & & 0,004 & & & $<0,001$ \\
\hline Superior a três quilos & $12(70,5)$ & $94(32,2)$ & & $27(73,0)$ & $175(30,7)$ & \\
\hline Não sabe informar & $3(17,7)$ & $41(14,0)$ & & $3(8,1)$ & $113(19,8)$ & \\
\hline Entre um a três quilos & $2(11,8)$ & $59(20,2)$ & & $5(13,5)$ & $143(25,1)$ & \\
\hline Sem perda de peso & - & $98(33,6)$ & & $2(5,4)$ & $139(24,4)$ & \\
\hline \multicolumn{7}{|l|}{ Utiliza mais de três } \\
\hline medicamentos diários & & & 0,795 & & & 0,506 \\
\hline $\operatorname{Sim}$ & $8(47,1)$ & $128(43,8)$ & & $25(67,6)$ & $354(62,1)$ & \\
\hline Não & $9(52,9)$ & $164(56,2)$ & & $12(32,4)$ & $216(37,9)$ & \\
\hline Mobilidade & & & & & & $<0,001$ \\
\hline $\begin{array}{l}\text { Deambula, não sai de } \\
\text { casa } \\
\text { Normal }\end{array}$ & $\begin{array}{l}3(17,6) \\
14,82,4)\end{array}$ & $\begin{array}{c}24(8,2) \\
268(91,8)\end{array}$ & 0,181 & $\begin{array}{l}10(27,0) \\
27(73,0)\end{array}$ & $\begin{array}{c}40(7,1) \\
530(92,9)\end{array}$ & \\
\hline $\begin{array}{l}\text { Lesões de pele ou } \\
\text { escaras }\end{array}$ & & & $<0,001$ & & & 0,195 \\
\hline $\operatorname{Sim}$ & $9(52,9)$ & $53(18,1)$ & & $10(27,0)$ & $105(18,5)$ & \\
\hline Não & $8(47,1)$ & $239(81,9)$ & & $27(73,0)$ & $465(81,5)$ & \\
\hline $\begin{array}{l}\text { Problemas } \\
\text { neurológicos } \\
\text { Demência ou depressão } \\
\text { grave }\end{array}$ & - & & 0,056 & & & $<0,001$ \\
\hline Demência ligeira & $3(17,6)$ & $14(4,8)$ & & $15(40,6)$ & $55(9,7)$ & \\
\hline Sem problemas & $14(82,4)$ & $14(4,8)$ & & $8(21,6)$ & $42(7,4)$ & \\
\hline psicológicos & & $264(90,4)$ & & $14(37,8)$ & $473(82,9)$ & \\
\hline $\begin{array}{l}\text { Passou por estresse ou } \\
\text { doença aguda }\end{array}$ & & & $\mathbf{0 , 0 0 7}$ & & & $<0,001$ \\
\hline Sim & $9(52,9)$ & $69(23,7)$ & & $32(86,5)$ & $248(43,5)$ & \\
\hline Não & $8(47,1)$ & $223(76,3)$ & & $5(13,5)$ & $322(56,5)$ & \\
\hline
\end{tabular}

*Teste qui-quadrado. 
$\mathrm{Na}$ comparação entre os idosos em relação às variáveis alimentares e de auto avaliação, os desnutridos apresentaram percentuais menores do número de refeições (1 refeições, 29,4\% vs 3,8\%), modo de se alimentar (não se alimentar sozinho/com dificuldade, $23,5 \%$ vs 4,1\%), diminuição da ingestão alimentar (grave e moderada, $100 \%$ vs 51,0\%), auto percepção da saúde (pior, 47,1\% vs $17,2 \%$ ) e problema nutricional (acredita estar desnutrido, 58,8\% vs 12,3\%) (Tabela 3).

Para as variáveis alimentares e de auto avaliação observou-se que as idosas desnutridas apresentaram menores percentuais no número de refeições (1 refeição, $13,5 \%$ vs 5,1\%), consomem duas ou mais porções diárias de frutas ou vegetais (não, 56,8\% vs 38,3\%), modo de se alimentar (não se alimenta sozinha/com dificuldade, $16,2 \%$ vs 4,1\%), diminuição da ingestão alimentar (grave e moderada, 78,4\% vs 62,8\%), auto percepção da saúde (pior, 62,2\% vs 21,6\%) e problema nutricional (acredita estar desnutrida, $51,4 \%$ vs 12,3\%) (Tabela 3 ).

Tabela 3 - Número e porcentagem de idosos segundo variáveis alimentares e percepção da saúde avaliadas pela Mini Avaliação Nutricional por sexo e estado nutricional. Superintendência Regional de Saúde de Uberaba, 2013

\begin{tabular}{|c|c|c|c|c|c|c|}
\hline \multirow[b]{2}{*}{ Variáveis } & \multicolumn{2}{|c|}{ Homens } & \multicolumn{4}{|c|}{ Mulheres } \\
\hline & $\begin{array}{c}\text { Desnutrição } \\
\text { n }(\%)\end{array}$ & $\begin{array}{c}\text { Risco de } \\
\text { desnutrição } \\
\text { n (\%) }\end{array}$ & $\mathbf{p}^{*}$ & $\begin{array}{c}\text { Desnutrição } \\
\text { n (\%) }\end{array}$ & $\begin{array}{c}\text { Risco de } \\
\text { desnutrição } \\
\text { n (\%) }\end{array}$ & $\mathbf{p}^{*}$ \\
\hline \multicolumn{7}{|l|}{ Alimentares } \\
\hline $\begin{array}{l}\text { Número de refeições } \\
1 \\
2 \\
3\end{array}$ & $\begin{array}{l}5(29,5) \\
3(17,6) \\
9(52,9)\end{array}$ & $\begin{array}{c}11(3,8) \\
111(38,0) \\
170(58,2)\end{array}$ & $<\mathbf{0 , 0 0 1}$ & $\begin{array}{c}5(13,5) \\
13(35,1) \\
19(51,4)\end{array}$ & $\begin{array}{c}29(5,1) \\
166(29,1) \\
375(65,8)\end{array}$ & 0,051 \\
\hline $\begin{array}{l}\text { Consome } 1 \text { porção diária de } \\
\text { leite ou derivados } \\
\text { Sim } \\
\text { Não }\end{array}$ & $\begin{array}{l}9(52,9) \\
8(47,1)\end{array}$ & $\begin{array}{l}158(54,2) \\
134(45,8)\end{array}$ & 0,925 & $\begin{array}{l}21(56,8) \\
16(43,2)\end{array}$ & $\begin{array}{l}345(60,5) \\
225(39,5)\end{array}$ & 0,650 \\
\hline $\begin{array}{l}\text { Consome } 2 \text { ou mais porções } \\
\text { semanais de leguminosas ou } \\
\text { ovos } \\
\text { Sim } \\
\text { Não }\end{array}$ & $\begin{array}{l}9(52,9) \\
8(47,1)\end{array}$ & $\begin{array}{c}209(71,5) \\
83(28,5)\end{array}$ & 0,101 & $\begin{array}{l}24(64,9) \\
13(35,1)\end{array}$ & $\begin{array}{l}433(76,0) \\
137(24,0)\end{array}$ & 0,129 \\
\hline $\begin{array}{l}\text { Consome carne, peixe ou aves } \\
\text { todos os dias } \\
\text { Sim } \\
\text { Não }\end{array}$ & $\begin{array}{l}9(52,9) \\
8(47,1)\end{array}$ & $\begin{array}{l}210(72,0) \\
82(28,0)\end{array}$ & 0,094 & $\begin{array}{l}26(70,3) \\
11(29,7)\end{array}$ & $\begin{array}{l}386(67,8) \\
184(32,2)\end{array}$ & 0,747 \\
\hline $\begin{array}{l}\text { Consome } 2 \text { ou mais porções } \\
\text { diária de frutas ou vegetais } \\
\text { Não } \\
\text { Sim }\end{array}$ & $\begin{array}{l}9(52,9) \\
8(47,1)\end{array}$ & $\begin{array}{l}156(53,5) \\
136(46,5)\end{array}$ & 0,969 & $\begin{array}{l}21(56,8) \\
16(43,2)\end{array}$ & $\begin{array}{l}218(38,3) \\
352(61,7)\end{array}$ & 0,026 \\
\hline $\begin{array}{l}\text { Quantos copos de líquidos } \\
\text { consomem diariamente } \\
<3 \text { copos } \\
3 \text { a } 5 \text { copos } \\
\geq 5 \text { copos }\end{array}$ & $\begin{array}{l}4(23,5) \\
5(29,4) \\
8(47,1)\end{array}$ & $\begin{array}{c}31(10,6) \\
87(29,8) \\
174(59,6)\end{array}$ & 0,247 & $\begin{array}{c}4(10,9) \\
15(40,5) \\
18(48,6)\end{array}$ & $\begin{array}{c}75(13,1) \\
208(36,5) \\
287(50,4)\end{array}$ & 0,851 \\
\hline $\begin{array}{l}\text { Modo de se alimentar } \\
\text { Não se alimentar sozinho } \\
\text { Com dificuldade } \\
\text { Sem dificuldade }\end{array}$ & $\begin{array}{c}1(5,9) \\
3(17,6) \\
13(76,5)\end{array}$ & $\begin{array}{c}5(1,7) \\
7(2,4) \\
280(95,9)\end{array}$ & 0,001 & $\begin{array}{c}1(2,7) \\
5(13,5) \\
31(83,8)\end{array}$ & $\begin{array}{c}12(2,2) \\
11(1,9) \\
547(95,9)\end{array}$ & $<0,001$ \\
\hline \multicolumn{7}{|l|}{ Auto avaliação } \\
\hline $\begin{array}{l}\text { Diminuição da ingesta } \\
\text { alimentar } \\
\text { Grave } \\
\text { Moderada } \\
\text { Sem diminuição }\end{array}$ & $\begin{array}{c}9(52,9) \\
8(47,1) \\
-\end{array}$ & $\begin{array}{c}38(13,0) \\
111(38,0) \\
143(49,0)\end{array}$ & $<0,001$ & $\begin{array}{l}16(43,3) \\
13(35,1) \\
8(21,6)\end{array}$ & $\begin{array}{l}102(17,9) \\
256(44,9) \\
212(37,2)\end{array}$ & 0,001 \\
\hline $\begin{array}{l}\text { Auto percepção da saúde } \\
\text { Pior } \\
\text { Não sabe } \\
\text { Igual } \\
\text { Melhor }\end{array}$ & $\begin{array}{l}8(47,1) \\
3(17,6) \\
5(29,4) \\
1(5,9)\end{array}$ & $\begin{array}{c}50(17,2) \\
32(10,9) \\
168(57,5) \\
42(14,4)\end{array}$ & 0,010 & $\begin{array}{c}23(62,2) \\
10(27,0) \\
3(8,1) \\
1(2,7)\end{array}$ & $\begin{array}{l}123(21,6) \\
81(14,2) \\
275(48,2) \\
91(16,0)\end{array}$ & $<0,001$ \\
\hline $\begin{array}{l}\text { Problema nutricional } \\
\text { Acredita estar desnutrido } \\
\text { Não sabe dizer } \\
\text { Acredita não ter problema }\end{array}$ & $\begin{array}{l}10(58,8) \\
5(29,4) \\
2(11,8)\end{array}$ & $\begin{array}{l}36(12,3) \\
49(16,8) \\
207(70,9)\end{array}$ & $<0,001$ & $\begin{array}{l}19(51,4) \\
10(27,0) \\
8(21,6)\end{array}$ & $\begin{array}{c}70(12,3) \\
103(18,1) \\
397(69,6)\end{array}$ & $<0,001$ \\
\hline
\end{tabular}

*Teste qui-quadrado. 


\section{DISCUSSÃO}

Os resultados demonstraram elevado déficit do estado nutricional dos idosos. $29,5 \%$ foram diagnosticados desnutridos ou sob risco de desnutrição. As prevalências de desnutrição e risco nutricional foram mais acentuadas nas idosas, principalmente acima de 70 anos de idade.

Os idosos desnutridos, como esperado em ambos os sexos, apresentaram piores parâmetros antropométricos, de saúde, alimentares e de auto avaliação da percepção da saúde e do estado nutricional quando comparados com os idosos sob risco de desnutrição.

Ao avaliar os parâmetros de diagnóstico do estado nutricional entre os idosos desnutridos e os sob risco de desnutrição, a redução do perímetro da panturrilha, perda de peso, diminuição da ingestão alimentar e o não consumo de frutas e vegetais apresentaram maiores percentuais.

Estudos nacionais de base populacional que avaliaram o estado nutricional de idosos residentes na comunidade e que utilizaram a MAN são escassos. Porém, pode-se citar o Health Well-Beingand Ageing (SABE) que avaliou 1.170 idosos residentes na cidade de São Paulo utilizando a MAN e observou prevalências de desnutrição e risco de desnutrição próximas aos achados nesse estudo $2,4 \%$ e $25,6 \%$, respectivamente 9 .

Ao comparar com estudos realizados em outros países que também utilizaram a MAN como critério de diagnóstico do estado nutricional, as prevalências de desnutrição e risco de desnutrição são diferentes devido aos critérios utilizados na inclusão dos idosos.

No estudo de coorte prospectivo realizado na província de Razavi Khorasan no Irã que incluiu 1.962 idosos ( $\geq 60$ anos) residentes em áreas rurais e urbanas, a prevalência de desnutrição foi de $12,0 \%$ e risco de desnutrição 45,3\%. ${ }^{7}$ Por outro lado, o estudo de Cuervo et al. ${ }^{17}$ observou a prevalência de desnutrição de $4,3 \%$ e risco de desnutrição de $25,4 \%$ em idosos residentes na Espanha.

No presente estudo observou-se diferenças em relação ao sexo sendo maior entre as mulheres indo ao encontro dos resultados do estudo Aging and Malnutrition in Elderly Lebanese que incluiu 1.200 idosos ( $\geq 65$ anos) residentes em sete áreas rurais no Líbano, demonstrando prevalência de maior desnutrição entre as mulheres quando comparadas com os homens $(9,1 \%$ vs $6,9 \%)$ assim como de risco de desnutrição (35,3\% vs $22,9 \%)$. Os autores discutem que tais diferenças encontradas foram decorrentes do fato de que mulheres eram altamente desfavorecidas sobre sua situação socioeconômica (nível de escolaridade baixo e renda baixa) e de saúde quando comparadas aos homens ${ }^{8}$.

Dentre os principais parâmetros de diagnóstico do estado nutricional entre os idosos desnutridos e sob risco de desnutrição observados (redução do perímetro da panturrilha, perda de peso, diminuição da ingestão alimentar e o não consumo de frutas e vegetais), acreditase haver uma interação entre eles e que a diminuição da ingestão alimentar possa desencadear os demais.

Os motivos que levam à diminuição da ingestão alimentar são vários e um deles frequentemente observado entre os idosos é a anorexia senil ${ }^{18}$. Landi et al. ${ }^{19}$ demonstraram, por meio de estudo longitudinal, que a anorexia é um fator de risco de mortalidade independente do baixo peso e de outros fatores clínicos confundidores.

A literatura aponta diversos fatores envolvidos na ocorrência da anorexia senil, tais como disfunções da mastigação e/ou deglutição, problemas de dentição, alteração de neurotransmissores envolvidos no controle do apetite e digestão, comorbidades e polifarmácia ${ }^{20,21}$. Além disso, depressão, fatores financeiros e sociais também podem estar envolvidos ${ }^{22}$.

A diminuição da ingestão alimentar, agravada pela presença de anorexia, leva à diminuição do peso corporal, alteração da composição corporal e mudança do comportamento alimentar. Portanto, ela explica em parte a baixa ingestão dos grupos alimentares fontes de proteína (leite, leguminosas e carnes) observado nos idosos desnutridos e sob risco de desnutrição.

Donini et al. ${ }^{23}$ estudaram idosos residentes em quatro cidades Italianas e observaram menor consumo de alimentos fontes de proteína entre aqueles que apresentaram anorexia quando comparados com os sem anorexia (ingestão de leite $<1$ vez ao dia $21,8 \%$ vs $15,3 \%$, carne vermelha $<4$ vezes na semana $44,8 \%$ vs $14,6 \%$, peixe menos que 3 vezes na semana $96,3 \%$ vs $56,4 \%$ e frango $<2$ vezes na semana 78,6 vs $37,3 \%$, respectivamente). A baixa ingestão das carnes no grupo que apresentou anorexia, segundo os autores, foi decorrente da dificuldade de mastigação, ajuda na compra e no preparo dos alimentos.

Além da diminuição do apetite que pode afetar a variabilidade da ingestão alimentar ${ }^{24}$, é importante ressaltar que esses grupos alimentares são perecíveis e não podem ser estocados por muito tempo no domicílio, exceto as carnes que podem ser congeladas. Assim, para tê-los disponíveis no seu dia a dia, os idosos precisam sair com frequência para comprá-los.

Por outro lado, alimentar-se de forma saudável e incorporar esses grupos alimentares é mais dispendioso e necessita de recursos financeiros adequados, o que muitas vezes pode ser uma barreira para idosos que dependem somente da aposentadoria. ${ }^{24}$

De acordo com os resultados obtidos, os idosos desnutridos e sob risco de desnutrição apresentaram massa muscular reduzida, avaliada pelo perímetro da panturrilha, considerado um indicador antropométrico sensível a alterações musculares ${ }^{25,26}$.

A redução da massa muscular durante o envelhecimento envolve fatores intrínsecos (genéticos, 
hormonais) e extrínsecos (alimentação, atividade física). ${ }^{27}$ Porém, a ingestão inadequada de proteína que fornecem os aminoácidos essenciais produz o catabolismo da proteína muscular e este, por sua vez, contribui na redução da massa muscular ${ }^{28}$.

O estudo longitudinal de Houston et al. ${ }^{29}$ demonstrou que os idosos no quinto quintil de ingestão de proteína perderam aproximadamente $40 \%$ menos massa magra total quando comparados com aqueles no primeiro quintil. Além disso, os autores sugerem que a baixa ingestão proteica pode ser um fator de risco modificável para sarcopenia. Em outro estudo longitudinal que envolveu 24.417 mulheres com idade entre 65 a 79 anos observou-se a associação entre alta ingestão de proteína com o baixo risco de fratura ${ }^{30}$. Rondanelli et al. ${ }^{31}$ observaram efeito benéfico no ganho de massa magra em idosos sarcopenicos que receberam suplementação de aminoácidos essenciais, vitamina $\mathrm{D}$ e exercício físico.

A sarcopenia é uma síndrome geriátrica caracterizada pela perda progressiva e generalizada da massa e da força muscular. ${ }^{32}$ A prevalência da sarcopenia segundo uma recente revisão sistemática realizada por Cruz-Jentoft et al. ${ }^{33}$ pode variar de $1 \%$ a $29 \%$ entre idosos que residem na comunidade. Ela tem sido associada com anorexia e quedas ${ }^{19}$ e declínio cognitivo ${ }^{34}$.

A importância da ingestão das proteínas constatada nos estudos citados anteriormente vai ao encontro dos especialistas da European Society for Clinical Nutritional and Metabolism. Em 2013, com o objetivo de preservar a massa muscular dos idosos, analisaram estudos prévios

e propuseram novas recomendações da quantidade de proteína diária. Eles recomendam de 1,0 a $1,2 \mathrm{~g} / \mathrm{kg}$ dia para os idosos saudáveis e na presença de doenças agudas/ crônicas de 1,2-1,5 g/kg dia ${ }^{35}$.

$\mathrm{O}$ presente estudo traz alguns pontos relevantes que devem ser considerados: a utilização da MAN um instrumento internacionalmente utilizado para a avaliação do estado nutricional proporcionando a comparação com outros estudos e a utilização do Mini Exame do Estado Mental ${ }^{13}$ como critério de seleção dos idosos minimizando o viés de memória. Embora exista limitação, o fato de as entrevistas terem sido realizadas com os idosos disponíveis nos dias das avaliações, não tínhamos informações em relação aos recursos disponíveis dos idosos para o acesso aos alimentos, os resultados não permitem a generalização a outros grupos de idosos.

\section{CONCLUSÃO}

O elevado déficit do estado nutricional, desnutrição e risco de desnutrição, observado nos idosos residentes nos municípios avaliados demonstra um problema de saúde pública. Espera-se que os achados desse estudo chamem a atenção de setores que trabalham com a integralidade da saúde dos idosos. Além disso, que nas diferentes práticas clínicas da saúde deem atenção para a avaliação global do estado nutricional de idosos. Pois, na maioria das vezes o idoso não dá a devida importância, não relata queixas referente a sua nutrição e que fazem toda diferença no sucesso de seu tratamento, saúde e na sua qualidade de vida.

Agradecimentos: Artigo parte do Projeto intitulado: "Perfil de Saúde da População Idosa dos Municípios da Gerência Regional de Saúde - Uberaba/ Minas Gerais, financiado pela Fundação de Apoio à Pesquisa de Minas Gerais (FAPEMIG) sob número: APQ-00995-11.

Participação dos autores: Renata Damião coordenou o projeto, realizou a análise e interpretação dos dados e redigiu o manuscrito. Joilson Meneguci participou da interpretação dos dados e redigiu o manuscrito. Álvaro da Silva Santos coordenou o projeto e redigiu o manuscrito. Alicia Matijasevich participou da análise, interpretação dos dados e redigiu o manuscrito. Paulo Rossi Menezes participou da análise, interpretação dos dados e redigiu o manuscrito.

\section{REFERÊNCIAS}

1. United Nations Population Fund (UNFPA), Help Age International. Ageing in the twenty-first century: a celebration and a challenge. New York; 2012 [cited 2015 July 04]. Available from: http://www.unfpa.org/sites/default/files/pubpdf/Ageing\%20report.pdf/Acesso: 04 Jul. 2015.

2. Instituto Brasileiro de Geografia e Estatística (IBGE). Censo demográfico (2010). IBGE; 2010 [citado nov. 2012]. Disponível em: http://www.censo2010.ibge.gov.br/sinopse/ webservice/.

3. Rolls BJ. Do chemosensory changes influence food intake in the elderly? Physiol Behav 1999; 66(2):193-7.

4. Freitas AF, Prado MA, Cação JC, Beretta D. Sarcopenia e estado nutricional de idosos: uma revisão da literatura. Arq Ciên Saúde. 2015;22(1):9-13. doi: 10.17696/23183691.22.1.2015.19.

5. Campos ACV, Ferreira EF, Vargas AMD, Albala C. Aging, Gender and Quality of Life (AGEQOL) study: factors associated with good quality of life. Health Qual Life
Outcomes. 2014;12(166):1-11. doi: 10.1186/s12955-0140166-4.

6. Palgi Y, Shrira A, Zaslavsky O. Quality of life attenuates age-related decline in functional status of older adults. Qual Life Res. 2015;24:1835-43. doi 10.1007/s11136-015-0918-6.

7. Aliabadi M, Kimiagar M, Ghayour-Mobarhan M, et al. Prevalence of malnutrition in free living elderly people in Iran: a cross-sectional study. Asia Pac J Clin Nutr. 2008;17(2):285-9. Available from: http://apjen.nhri.org.tw/ server/APJCN/17/2/285.pdf.

8. Boulos C, Salameh P, Barberger-Gateau P. Factors associated with poor nutritional status among community dwelling Lebanese elderly subjects living in rural areas: results of the AMEL study. J Nutr Health Aging. 2014;18(5):487-94. doi: 10.1007/s12603-013-0436-6.

9. Ferreira LS, Amaral TF, Marucci, MFN, Nascimento LFC, Lebrão ML, Duarte YAO. Undernutrition as a major risk factor for death among older Brazilian adults in the communitydwelling setting: SABE survey. Nutrition. 2011;27(10):101722. doi: 10.1016/j.nut.2010.11.008. 
Damião R, et al. Estado Nutricional de idosos residentes nos municípios da Superintendência Regional.

10. Boulos C, Salameh P, Barberger-Gateau P. The AMEL study, a cross sectional population-based survey on aging and malnutrition in 1200 elderly Lebanese living in rural settings: protocol and sample characteristic. BMC Public Health. 2013;573:2-13. doi: 10.1186/1471-2458-13-573.

11. Guigoz Y, Lauque S, Vellas BJ. Identifying the elderly at risk for malnutrition the mini nutritional assessment. Clin Geriatr Med. 2002;18(4):737-57. https://doi.org/10.1016/ S0749-0690(02)00059-9.

12. Barone L, Milosavljevic M, Gazibarich B. Assessing the older person: Is the MNA a more appropriate nutritional assessment tool than the SGA? J Nutr Health Aging. 2003;7(1):13-7.

13. Folstein MF, Folstein SE, Mchugh, PR. "Mini-mental state". A practical method for grading the cognitive state of patients for the clinician. J Psychiatr Res. 1975;12(3):189-98. https:// doi.org/10.1016/0022-3956(75)90026-6.

14. Bertolucci PHF, Brucki SMD, Campacci SR, et al. O MiniExame do Estado Mental em uma população geral: impacto da escolaridade. Arq Neuro-Psiquiatr. 1994;52(1):1-7. http:// dx.doi.org/10.1590/S0004-282X1994000100001.

15. Lohman TG, Roche AF, Martorell R. Anthropometric Standardization Reference Manual. Champaign: Human Kinetics Books; 1988.

16. Mini Nutritional Assessment [cited 201 July]. Available from: http://www.mna-elderly.com/forms/MNA_portuguese.pdf.

17. Cuervo M, Ansorena D, Martinez-Gonzalez MA, García A, Astiasarán I, Martínez JA. Impact of global and subjective mini nutritional assessment (MNA) questions on the evaluation of the nutritional status: the role of gender and age. Arch Gerontol Geriatr. 2009;49(1):69-73. doi: 10.1016/j. archger.2008.05.003.

18. Visvanathan R. Anorexia of aging. Clin Geriatr Med. 2015;31(3):417-27. doi: 10.1016/j.cger.2015.04.012.

19. Landi F, Liperoti R, Russo A, Giovannini S, Tosato M, Barillaro C, et al. Association of anorexia with sarcopenia in a community-dwelling elderly population: results from the ilSIRENTE study. Eur J Nutr. 2013;52(3):1261-8. doi: 10.1007/s00394-012-0437-y.

20. Hays NP, Robersts SB. The anorexia of aging in humans. Physiol Behav. 2006;88(3):257-66. doi: 10.1016/j. physbeh.2006.05.029.

21. Morley J, Anker SD, Evans WJ. Cachexia and aging: an update based on the Fourth International Cachexia Meeting. J Nutr Health Aging. 2009;13(1):47-55.

22. Soenen S, Chapman IM. Body weight, anorexia, and undernutrition in older people. JAMDA. 2013;14(9):642-8. doi: 10.1016/j.jamda.2013.02.004.

23. Donini LM, Dominguez LJ, Barbagallo M, Savina C, Castellaneta E, Cucinotta D, et al. Senile anorexia in different geriatric settings in Italy. JNHA. 2011;15(9):775-81.

24. Dean M, Raats MM, Grunert KG, Lumbers M. The food in later life team. Factors influencing eating a varied diet in old age. Public Health Nutr. 2009;12(12):2421-7. doi: 10.1017/ S1368980009005448.

25. World Health Organization (WHO). Physical status: the use and interpretation of anthropometry. Geneva; 1995. Available from: https://www.who.int/childgrowth/publications/ physical_status/en/.

26. Kawakami R, Murakami H, Sanada K, Tanaka N, Sawada SS, Tabata I, et al. Calf circumference as a surrogate marker of muscle mass for diagnosing sarcopenia in Japanese men and women. Geriatr Gerontol Int. 2015;15(8):969-76. doi: 10.1111/ggi.12377.

27. Waters DL, Baumgartner RN. Sarcopenia and Obesity. Clin Geriatr Med. 2011;27(3):401-21. doi: 10.1016/j. cger.2011.03.007.

28. Paddon-Jones D, Short KR, Campbell WW, Volpi E, Wolfe RR. Role of dietary protein in the sarcopenia of aging. Am J Clin Nutr. 2008;87(Suppl):1562S-6S. doi: 10.1093/ ajcn/87.5.1562S

29. Houston DK, Nicklas BJ, Ding J, Harris TB, Tylavsky AF, Newman AB, et al. Dietary protein intake is associated with lean mass change in older, community-dwelling adults: the Health, Aging, and Body Composition (Health ABC) Study. Am J Clin Nutr. 2008;87(1):150-5. doi: 10.1093/ ajcn/87.1.150.

30. Beasley JM, LaCroix AZ, Neuhouser ML, Huang Y, Tinker L, Woods N, Michael Y, Curb JD, Prentice RL. Protein intake and incident frailty in the Women's Health Initiative Observational Study. J Am Geriatr Soc. 2010;58(6):106371. doi: 10.1111/j.1532-5415.2010.02866.x.

31. Rondanelli M, Klersy C, Terracol G, Talluri J, Maugeri R, Guido D, et al. Whey protein, amino acids, and vitamin D supplementation with physical activity increases fat-free mass and strength, functionality, and quality of life and decreases inflammation in sarcopenic elderly. Am J Clin Nutr. 2016;103(3):830-40. doi: 10.3945/ajcn.115.113357.

32. Rizzoli R, Stevenson JC, Bauer JM, van Loon LJC, Walrand $\mathrm{S}$, Kanis JA, et al.The role of dietary protein and vitamin D in maintaining musculoskeletal health in postmenopausal women: A consensus statement from the European Society for Clinical and Economic Aspects of Osteoporosis and Osteoarthritis (ESCEO). Maturitas. 2014;79(1):122-32. doi: 10.1016/j.maturitas.2014.07.005 0378-5122.

33. Cruz-Jentoft AJ, Landi F, Schneider SM, Zúñiga C, Arai $\mathrm{H}$, Boirie $\mathrm{Y}$, et al. Prevalence of and interventions for sarcopenia in ageing adults: a systematic review. Report of the International Sarcopenia Initiative (EWGSOP and IWGS). Age Ageing. 2014;43(6):748-59. doi: 10.1093/ ageing/afu115.

34. Nishiguchi S, Yamada M, Shirooka H, Nozaki Y, Fukutani N, Tashiro Y, et al. Sarcopenia as a risk Factor for cognitive deterioration in community-dwelling older adults: a 1-Year Prospective Study. JAMDA. 2016;17(4):372.e5-372.e8. doi: 10.1016/j.jamda.2015.12.096.

35. Deutz NE, Bauer JM, Barazzoni R, Biolo G, Boirie Y, BosyWestphal A, et al. Protein intake and exercise for optimal muscle function with aging: recommendations from the ESPEN Expert Group. Clin Nutr. 2014;33(6):929-36. doi: 10.1016/j.clnu.2014.04.007.

Recebido: 07.01.19

Aceito: 08.08.19 